\title{
Metastatic renal cell carcinoma mimicking pleural mesothelioma
}

\author{
D R TAYLOR, W PAGE, D HUGHES, G VARGHESE \\ From the Departments of Medicine and Pathology, The Queen's University of Belfast
}

Renal cell carcinoma is renowned for its propensity to metastasise in unusual ways. While the thorax is the most frequently affected site, pleural lesions are uncommon, and metastasis is usually associated with parenchymal lung lesions. ${ }^{1}$ We report a case in which pleural metastatic disease was the sole intrathoracic abnormality and the clinical, radiological, and pathological features were identical to those of pleural mesothelioma.

\section{Case report}

A 67 year old man presented with a six week history of dry cough, increasing dyspnoea and left sided pleuritic chest pain. He had been a lifelong smoker ( 40 pack years) and had been exposed to asbestos 25 years earlier while working as a boiler engineer. At the age of 56 years a right sided renal cyst had been drained surgically. He denied any urinary symptoms.

Physical examination showed an obese $(85 \mathrm{~kg})$, afebrile man with signs of a left sided pleural effusion. A firm, nontender mass measuring $6 \mathrm{~cm}$ in diameter was felt in the left hypochondrium. Examination of the urine showed numerous pus cells but no red cells.

The effusion was aspirated and pleural biopsy specimens were obtained, but cytological examination of the fluid failed to reveal malignant cells. The chest radiograph, taken after aspiration (fig 1), showed a lobulated pleural opacity extending into the paratracheal area and the presence of residual fluid. Computed tomography of the thorax (fig 2) and abdomen showed appearances suggesting a diagnosis of mesothelioma or metastatic disease; no pleural plaques were identified. Abdominal scans indicated a solid rather than cystic mass arising from the parenchyma of the left kidney. The appearances were typical of a renal cell carcinoma.

Reaccumulation of pleural fluid was managed by temporary insertion of an intercostal drain and tetracycline pleurodesis. The patient remained symptomatically stable for four months, but then deteriorated and died in respiratory failure.

At necropsy the left pleural space was completely obliterated by white fleshy tumour, encasing the lung and penetrating the pericardium and diaphragm. The macroscopic appearances were indistinguisable from those of pleural mesothelioma. In addition, the tumour had extended along the track of previous intercostal drainage, ending in a $4 \mathrm{~cm}$ lobulated subcutaneous mass. No other discreet metastatic

Address for reprint requests: Dr G Varghese, Level 8, Belfast City Hospital, Belfast BT9 7AB. lesion was found in the lungs or at the hila. Histological examination showed vacuolated polygonal cells diagnostic of renal cell carcinoma. Immunocytochemical studies confirmed the tumour to be renal in origin and excluded the possibility of mesothelioma. Positive results on testing with epithelial membrane antigen, low weight cytokeratin (kidney associated epithelia), vimentin, and periodic acid-Schiff indi-

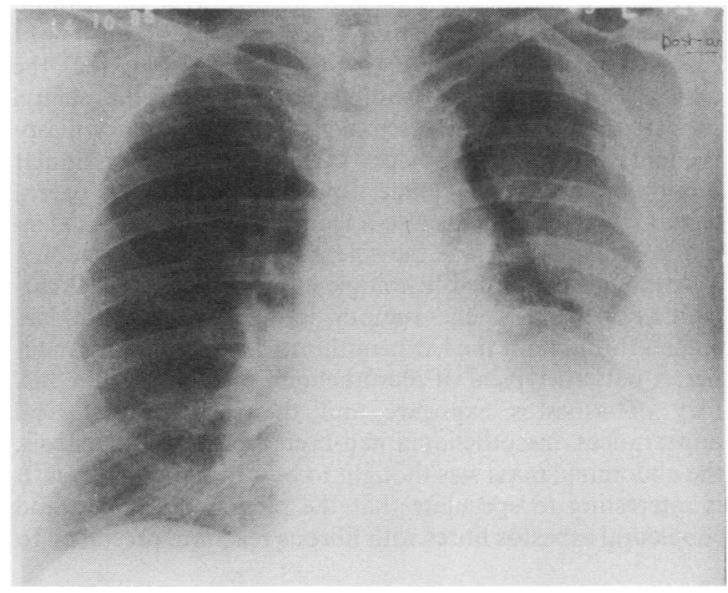

Fig 1 Chest radiograph taken after aspiration of the left sided pleural effusion, showing the lobulated pleural opacity extending into the paratracheal area.

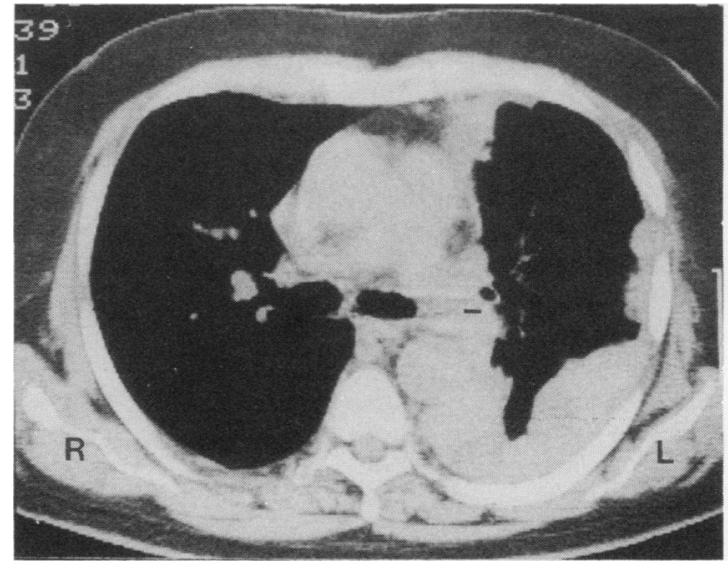

Fig 2 Computed tomography scan of the thorax, suggesting a diagnosis of mesothelioma or metastatic disease. 
cated a renal origin. Negative results with $52 \mathrm{kD}$ cytokeratin and MHFG2 excluded a primary mesothelioma. ${ }^{2}$

In the right lung the pleura was grossly thickened, although no plaques were seen. Asbestos fibres, mild fibrosis, and alveolar wall thickening, diagnostic of early asbestosis, were widely distributed subpleurally. In the abdomen the presence of a large renal cell carcinoma arising from the left kidney was confirmed, and in the right kidney a single $0.5 \mathrm{~cm}$ metastatic deposit was found.

\section{Discussion}

The thorax is the most common site for metastasis from a renal cell carcinoma, being affected in up to $55 \%$ of cases at necropsy. Multiple pulmonary nodules are most frequently found, but single nodules, lymphangitis carcinomatosa, hilar and mediastinal adenopathy, endobronchial obstruction, and thoracic wall lesions also occur. ${ }^{134}$

Although uncommon, pleural lesions with or without effusions are by no means rare, occurring in about $4 \%$ of cases. What is unusual about the present case is that the clinical behaviour and pathological features of the pleural metastasis so strikingly resembled that of a primary mesothelioma. We can find only one other report in which similar observations have been made. ${ }^{5}$ In that case, however, parenchymal lung disease was also a feature. In our patient extensive pleural tumour was the sole intrathoracic abnormality.

Although confirmed histologically to be a renal cell carcinoma metastasis, the tumour in the present case had spread throughout the left hemithorax in a contiguous manner, a pattern typical of mesothelioma. Because of the history of asbestos exposure and the initial radiological appearances, mesothelioma had been the clinical diagnosis; the abdominal mass was thought to be a benign renal cyst. It is interesting to speculate that the pleural thickening and subpleural asbestos fibres with fibrous reaction, presumed to be bilateral in their distribution, might have had an ime portant effect on tumour behaviour after the establishmen of a single pleural metastasis.

One other feature makes this case unusual. The spread of tumour along the track of a previous intercostal drain in $\frac{0}{-}$ sertion is characteristic of mesothelioma and has been obs served after diagnostic thoracotomy, ${ }^{6}$ but has only rarel $\vec{b}$ been reported in cases of renal cell carcinoma after needle biopsy. $^{7}$

Finally, renal cancer has been found to occur at twice the anticipated rate in a large series of asbestos expose workers. $^{8}$ Whether in our case asbestos exposure was of aetiological significance cannot be established. The case is reminder, however, that asbestos is a possible cause of somer non-respiratory tract tumours.

\section{References}

1 Bennington JL, Kradjian RM. Distribution of metastases from renal cell carcinoma. In: Bennington JL, Kradjian RM, eds을 Renal carcinoma. Philadelphia: WB Saunders, 1967:156-7.

2 Bolen JW, Hammer SP, McNutt C. Reactive and neoplastic sero sal tissue. Am J Surg Pathol 1986;1:34-47.

3 Kutty K, Varkey B. Incidence and distribution of intrathoracic metastases from renal cell carcinoma. Arch Intern Medo 1984;144:273-6.

4 Riches EW. Tumours of the kidney and ureter. In: DW Smithers) ed. Neoplastic disease at various sites. Vol 5. Edinburgh: ES Livingstone, 1964:72-85.

5 Latour A, Shulman HS. Thoracic manifestations of renal cell car cinoma. Radiology 1976;121:43-8.

6 Shearin GC, Jackson D. Malignant pleural mesotheliomareport of 19 cases. J Thoracic Cardiovasc Surg 1976;71:621-6థ

7 Bush WH, Burnett LL, Gibbons RP. Needle track seeding of renal cell carcinoma. Am J Roentgenol 1977;129:725-7.

8 Selikoff IJ, Hammond EC, Seidman H. Mortality experience of insulation workers in the United States and Canada 1943-1976. Ann NY Acad Sci 1979;330:91-116. 